Georgia State University

ScholarWorks @ Georgia State University

\title{
Reading Skill in Adult Survivors of Childhood Brain Tumor: A Theory-Based Neurocognitive Model
}

\author{
Kristen M. Smith \\ Tricia Z. King \\ Georgia State University, tzking@gsu.edu \\ Reema Jayakar \\ Robin Morris \\ Georgia State University, robinmorris@gsu.edu
}

Follow this and additional works at: https://scholarworks.gsu.edu/psych_facpub

Part of the Psychology Commons

\section{Recommended Citation}

Smith K.M., King T.Z., Jayakar, R., \& Morris, R.D. (in press). Reading skill in adult survivors of childhood brain tumor: A theory-based neurocognitive model using DTI. Neuropsychology, Volume(Number), pages. DOI:

This Article is brought to you for free and open access by the Department of Psychology at ScholarWorks @ Georgia State University. It has been accepted for inclusion in Psychology Faculty Publications by an authorized administrator of ScholarWorks @ Georgia State University. For more information, please contact scholarworks@gsu.edu. 


\title{
Running head: MODEL OF READING IN BRAIN TUMOR SURVIVORS
}

Reading skill in adult survivors of childhood brain tumor: A theory-based neurocognitive model Kristen M. Smith, Tricia Z. King, Reema Jayakar, Robin D. Morris

Georgia State University

\begin{abstract}
Objective: This study investigated the relationship between word reading and white matter (WM) integrity within a neuroanatomical-based reading system comparing adult survivors of childhood brain tumors and controls. It was predicted that the association between WM integrity and word reading would be mediated by processing speed, and this indirect effect would be moderated by group. Method: Thirty-seven adult survivors of childhood brain tumor and typically developing adults participated (age $M=24.19 \pm 4.51$ years, $62 \%$ female). DTI Tractography identified the WM tract for three of the reading system connections: inferior fronto-occipital fasciculus (IFOF), arcuate fasciculus (AF), and parietotemporaloccipitotemporal connection (PT-OT). Results: Fractional anisotropy values (FA) of the PT-OT tract were significantly correlated with word reading in survivors and controls $(\mathrm{r}=.45, .58$, respectively; $\mathrm{p}<.05)$ while IFOF values were associated with reading in survivors only $(\mathrm{r}=.59$, $\mathrm{p}<.01)$. Further, the moderated mediated model was significant for PT-OT and IFOF, such that the indirect effect of processing speed was only present for survivors (CI: PT-OT: 2.90, 28.41, IFOF: 2.92, 40.17). Conclusion: Results suggest the tracts emerging from the occipitotemporal area are a critical component of the reading system in adults. The finding that processing speed
\end{abstract}


was the mechanism by which WM was associated with reading in survivors is in alignment with the developmental cascade model. Current findings bolster the existing theory-based models of reading using innovative diffusion tensor imaging and moderated mediation statistical neurodevelopmental model, establishing the role of processing speed and specific WM pathway integrity in word reading skill.

Key words: Diffusion tensor tractography; Reading; Pediatric brain tumor survivorship; Long term; Processing speed 


\section{Model of Reading in Brain Tumor Survivors}

With more sophisticated medical technology to diagnose and treat brain tumors, survivors are living longer than ever before providing impetus to study long-term outcomes. Cognitive dysfunction can be substantial and 40 to 100 percent of long term survivors experience some type of cognitive dysfunction (Glauser \& Packer, 1991; Mulhern \& Palmer, 2003). However, cognitive difficulties are not always apparent immediately after treatment. In fact, many deficits do not appear until years after treatment is completed. Longitudinal studies have shown that survivors develop more slowly than their peers in verbal, nonverbal, and general intelligence (Palmer, 2008). These learning difficulties appear to be due to a reduced rate of skill acquisition rather than a loss of previously learned skills (Mabbott, Penkman, Witol, Strother, \& Bouffet, 2008; Palmer et al., 2001). Therefore, a survivor may continue to learn and incorporate new information, albeit at a slower rate; hence, the performance of the survivor relative to same age peers grows increasingly discrepant.

Although measures of intelligence provide an adequate measure of general cognition, more research is needed on more specific functional impairments such as reading ability that affect everyday life in order to plan specific interventions. Reading achievement appears particularly vulnerable to disease and treatment risk factors in brain tumor survivors. Several studies have found decoding scores for real words to be below normative values after treatment (Beebe et al., 2005; Kieffer-Renaux et al., 2000; Reddick et al., 2003; Reeves et al., 2006; Robinson et al., 2010). Studies examining short term survivorship (2 years or less post treatment) suggest decoding vulnerability in children treated with radiation therapy and also in those treated with surgery only (Beebe, et al., 2005; Reeves, et al., 2006). Furthermore, longitudinal data indicate slower reading skill development in survivors over time, with decoding scores on 
average remaining below normative scores, signifying that survivors have more delays in the rate at which they are developing reading skills (Mabbott, et al., 2008; Mulhern et al., 2005; Reddick, et al., 2003). A more recent study observed slower learning of reading decoding skills in survivors (Conklin, Li, Xiong, Ogg, \& Merchant, 2008), and also found that while standard scores for reading declined over time (median follow up time of 60 months), scores for spelling and math achievement remained at par with peers. These findings suggested that reading was more prone to difficulties than other areas of achievement in a group of childhood survivors of ependymoma brain tumor treated with radiation therapy.

While the aforementioned research suggests that reading development is disrupted across tumor types and treatment, reading has primarily been examined with regard to short term outcomes. Extended long term outcome studies, decades after diagnosis are scarce. Thus, relatively little is known about reading skills in long term survivors longer than 10 years since diagnosis. This study focuses on survivorship 17 years since diagnosis, on average, and young adults at an average age of examination of 25 years.

Understanding why survivors tend to struggle with learning and advancing reading skills is a fundamental step toward developing effective interventions. In survivors, academic achievement is considered a distal marker of a set of underlying deficits in core cognitive skills such as processing speed, attention, and memory (Mabbott et al., 2005; Mulhern, et al., 2005; Palmer, 2008). In Palmer's (2008) developmental cascade model of the neurodevelopmental impacts of brain tumors, disease and treatment risk factors were proposed to be associated with broad outcomes of academic achievement and intelligence. She further theorized that these risk factors are associated with broad outcomes through their impact on underlying core cognitive 
functions. The mechanistic role of core cognitive skills in this model, and the underlying neural correlates as they relate to achievement, has not been adequately studied.

Information processing speed is hypothesized to be one of the core cognitive skills associated with automatic reading skills and reading fluency. Information processing speed refers to the efficiency of processing simple cognitive or perceptual information (Palmer, 2008). In the general population, reading fluency difficulties are often accompanied by slower processing speed (Catts, Gillispie, Leonard, Kail, \& Miller, 2002; Shanahan et al., 2006). In brain tumor survivors, difficulties in processing speed have been observed in individuals with a variety of treatment regimens (i.e. surgery only, radiation and surgery, etc.). Processing speed has been identified as a possible precursor to broader cognitive dysfunction in survivors (Palmer, 2008), and it is hypothesized to be the first deficit to arise after treatment, and persists over time (Mabbott, et al., 2008). Furthermore, speed has been shown to be slowed even when deficits in other core skills have not been found (Briere, Scott, McNall-Knapp, \& Adams, 2008; Mabbott, et al., 2008). As processing speed is known to be associated with many complex cognitive processes, it was hypothesized that it is a precursor to potential reading difficulties in a population such as brain tumor survivors who experience global neural and cognitive insults due to various disease and treatment related factors.

In summary, survivors of childhood brain tumors experience reading difficulties reflecting slower rates of learning and automatization which may be related to slower processing speed. This theory-driven study aims to examine factors related to reading outcome and test a neurodevelopmental model of reading outcome in long term survivors. In addition, given the neurobiological effects of brain tumors and their treatment, the neural underpinnings related to reading were important to integrate into these existing models. 
Neurological insult in childhood, can negatively affect the development of white matter and further impact cognitive function (Cascio, Gerig, \& Piven, 2007). From fMRI studies, it has been learned that reading is a complex cognitive skill involving the coordination of multiple regions of the brain. Studies of word decoding have established a reliable network of left hemisphere brain regions responsible for skilled reading. This network consists of an anterior region in the inferior frontal gyrus (IFG), a dorsal posterior region in the parietotemporal area (PT), and a ventral posterior region in the occipitotemporal area (OT) consisting of the visual word form area in the fusiform gyrus (Pugh et al., 2001; B. A. Shaywitz et al., 2002). The parietotemporal region in particular appears to work in concert with the IFG to command early development of word decoding (Booth et al., 2001; Pugh et al., 2010), while further learning is facilitated by communication between the occipitotemporal area and IFG. The occipitotemporal area becomes central as proficiency in word decoding increases and this area is particularly attuned to skilled and fluent reading (B. A. Shaywitz et al., 2007; S. E. Shaywitz \& Shaywitz, 2008). Given the occipitotemporal area's facilitation of speeded, fluent reading, factors that impact processing speed may primarily impact the development of reading in this region.

Studies examining white matter structure and reading in typically developing participants and those with dyslexia have found support for the disruption of posterior reading systems in poor readers (Beaulieu et al., 2005; Deutsch et al., 2005; Klingberg et al., 2000; Niogi \& McCandliss, 2006). They have also found negative correlations between white matter microstructure in the corpus callosum and reading skill, which is hypothesized to reflect reduced lateralization in poor readers (Frye et al., 2008; Odegard, Farris, Ring, McColl, \& Black, 2009). However, little work has directly examined white matter tracts thought to belong to the hypothesized reading system. 
Previous work in brain tumor survivors has found associations between white matter structure and cognitive function and reading skill using whole brain volumetric or diffusion tensor imaging (DTI) voxel-based exploratory approaches (Palmer et al., 2010; Reddick, et al., 2003). However, DTI tractography allows for the more direct tracking and measurement of connections between brain regions (Mori \& van Zij1, 2002) and quantifies the white matter microstructure that is not apparent on traditional MRI. The ability to more directly examine connections between cortical functional reading related regions in the brain is crucial for identifying possible areas of poor neural communication in survivors. Therefore, it is important to examine whether and to what extent the neuroanatomical reading system has been disrupted and how this impacts reaching achievement outcomes for these survivors. Understanding the cognitive mechanisms by which white matter dysruption is associated with reading vulnerability in survivors will build upon and provide alternative evidence that strengthens existing models.

Aim 1: To examine word reading and the white matter tract microstructure connecting the primary cortical regions involved in skilled reading. It is planned to separately examine, (1) inferior frontal gyrus to parietotemporal area (IFG- PT), (2) inferior frontal gyrus to occipitotemporal area (IFG-OT), and (3) parietotemporal area to occipitotemporal area (PT-OT). It was hypothesized that both the survivor group and the healthy control group would have significant positive relationships between white matter integrity in each of the three measured tracts and word reading skill.

Aim 2: To test a moderated mediation model of the association between white matter integrity in each of the 3 tracts and word reading being mediated by processing speed. It is hypothesized that the indirect effect of processing speed as the mediator would be moderated by group (survivors vs. controls). 
Secondary analyses included testing the specificity of the model to reading and related white matter tracts by testing the model with skilled motor speed as the dependent variable in place of reading, as well as testing the model with a non-reading related tract (the cortico-spinal tract) as the independent variable.

\section{Method}

\section{Participants}

Nineteen adult survivors of childhood brain tumor and 19 demographically matched control participants took part in this study. The study was approved by local institutional review board and all participants signed informed consent. Brain tumor survivors were recruited from two sources using opt-in letters mailed to survivors identified by: 1) a previous longitudinal study, in which they participated as children 2) a local children's hospital. In these analyses, survivors were included if they were a native English speaker, demonstrated adequate vision, scan was free of metal artifact or distortion in left hemisphere and left hemisphere free of major abnormality near tested tracts and ROIs or resection site near tested tracts and ROIs. SCID (Structured Clinical Interview for DSM-IV-TR Axis 1) indicated all survivors were free of current psychopathology with the exception of one survivor who met diagnostic criteria for Dysthymia (First, Spitzer, Gibbon, \& Williams, 2002). Data from one survivor were excluded due to extreme scores on the neuropsychological measures, and deemed a multivariate outlier. Thus 18 survivors were included in analyses.

Nineteen healthy control participants were chosen to match the survivor group by age, sex, ethnicity, socioeconomic status, and level of education. The healthy control group was recruited from the local university's psychology department research subject pool, friends of 
survivors, and community sample fliers. Inclusion criteria for control participants included English as their native language and SCID interviews indicating free of current psychopathology.

The final sample of 37 participants (62\% female) had a mean age at the time of study participation of 24.19 years $(\mathrm{SD}=4.51$, range $=19.42$ to 40.67$)$ (see Table 1$)$. Participants had completed an average of 14.16 years $(\mathrm{SD}=1.52)$ of education. The Wechsler Abbreviated Scale of Intelligence (WASI) (Wechsler, 1999) four subtest version was used to measure intelligence. The Hollingshead Four Factor Index of Social Status (Hollingshead, 1975) was used to calculate socioeconomic status (SES) of each participant (scores of 1, 2, or 3 out of 5 were grouped as "high" SES). Seventy-nine percent of the sample was grouped as high socioeconomic status. Twenty-two percent of participants identified as African-American, 3\% as Asian, 57\% Caucasian, 8\% Hispanic, and 3\% mixed ethnicities. Groups were dichotomized into the majority ethnicity (Caucasian) and other ethnicities to test group differences. Groups were not significantly different on any demographic variable (see Table 1).

Survivors were on average 7.22 (4.57 SD) years at diagnosis (range 1-17 years) and 17.13 (5.43 SD) years post-diagnosis (range 5-24 years) at time of evaluation. Survivor tumor pathologies included: medulloblastoma (6), pineoblastoma (1), astrocytoma (9), ganglioglioma (1), and craniopharyngioma (1). Tumors were located in the parietal lobe (1), occipital lobe (1), optic nerve (2), pituitary (1), and posterior fossa (13). In terms of treatment, all survivors had neurosurgery to remove the tumor, 8 participants were treated with both radiation and chemotherapy, 1 participant treated with chemotherapy, and 1 treated with radiation therapy, and 6 survivors were also diagnosed with hydrocephalus.

\section{Measures}


Reading Achievement. Reading achievement was examined with the Letter-Word Identification (LWID) subtest of the Woodcock Johnson Tests of Achievement III (Woodcock, McGrew, \& Mather, 2001b). LWID is a measure of real word decoding. For this task, participants are presented with written words to read orally and the score is based on correct pronunciation (Woodcock, McGrew, \& Mather, 2001a). This measure has been utilized to quantify reading ability in survivors of childhood brain tumors, and children with dyslexia, and has been linked to white matter measures over a range of ability levels (Frye et al., 2010). LWID z-scores computed from normative data (Woodcock, et al., 2001b) were used as the primary dependent variable.

Information Processing Speed. The Symbol Digit Modalities Test (SDMT) Oral Version was used in this study as the measure for information processing speed. The SDMT Oral Version is a perceptual task that unlike many tests of processing speed, does not involve a motor component requiring fine visuo-motor coordination and speed. Instead it is based on a verbal response, allowing a more pure measure of information processing speed, as opposed to motor speed. The SDMT involves converting simple meaningless geometric designs into oral number responses according to a key that matches each symbol to a number (A. Smith, 1982). The participant is given a 90 second time limit to complete as many items as they can. The score is based on the number of items correctly completed within the time limit. The SDMT has been used extensively as a measure of processing speed in a range of populations, including various types of brain disorders (Christodoulou et al., 2003; Parmenter, Weinstock-Guttman, Garg, Munschauer, \& Benedict, 2007; Perrine et al., 1995). Oral SDMT z-scores computed from normative data (A. Smith, 1982) were used for the current study. 
Skilled Motor Speed. Skilled motor speed was used as the control task because while it is an important cognitive outcome in brain tumor survivors, it was not hypothesized to be strongly related to the white matter tracts tested. It was measured using the Grooved Pegboard test dominant hand score (Ruff \& Parker, 1993). This task requires the participant to fit key-holeshaped pegs into holes in a pegboard using only one hand at a time, going as quickly as possible. The raw score is the amount of time in seconds that it takes the participant to put pegs into all the holes on the board. Z- scores computed from normative data (Bornstein, 1985) were used in the current study.

White Matter Microstructure. Diffusion tensor imaging (DTI) is a noninvasive, in vivo technique used to indirectly measure the quality of white matter structure based on the diffusion of water within tissue. DTI is sensitive to changes in white matter due to injury, damage or development (Assaf \& Pasternak, 2008). Among bundles of myelinated axons, water diffuses along the direction of the axon in an anisotropic manner (Cascio, et al., 2007) which is quantified in this study using fractional anisotropy (FA), which is an indirect normalized measure of directional diffusion. High anisotropy suggests fast water diffusivity parallel to the fibers, and slow diffusivity perpendicular to the fibers (Assaf \& Pasternak, 2008). The FA index ranges between 0 and 1 with 1 suggesting maximal directional diffusion and 0 suggesting isotropic diffusion. However as an indirect and nonspecific measure, FA can be influenced by other cellular properties such as inflammation, axon density, axon diameter, and crossing fibers (Tournier, Mori, \& Leemans, 2011; Wheeler-Kingshott \& Cercignani, 2009). Thus, changes in these cellular properties rather than white matter integrity may also lead to changes in FA and differences should be interpreted with caution. Given that the brain is a complex system, 
however, DTI is a manageable non-invasive tool for the purpose of macroscopic white matter comparisons (Tournier, et al., 2011).

Scan Acquisition. A Siemens Trio 3T scanner with a standard RF 12 channel head coil was used to obtain DTI and anatomical data. A 30 direction single shot spin echo diffusionweighted sequence with 60 contiguous axial slices interleaved and 2 non-diffusion weighted images was acquired with $2 \times 2 \times 2 \mathrm{~mm}$ resolution and coverage of the whole head (b value $\left.=1000 \mathrm{~s} / \mathrm{mm}^{2}, \mathrm{TE} / \mathrm{TR}=90 \mathrm{~ms} / 7700 \mathrm{~ms}, \mathrm{FOV}=204 \mathrm{~mm}, \mathrm{GRAPPA}=2\right) .3 \mathrm{D}$ T1-weighted images were used for anatomical registration (176 contiguous sagittal slices, $\mathrm{TR}=2250 \mathrm{~ms}$, $\mathrm{TE}=3.98 \mathrm{~ms}$, flip angle $=9$ degrees, voxel $=1.0 \times 1.0 \times 1.0 \mathrm{~mm})$.

Probabilistic Tractography. Probabilistic tractography was used to identify the 3 hypothesized tracts between the functional reading-related regions. FDT 2.0 (FMRIB's Diffusion Toolbox) part of FSL 4.0 was utilized for diffusion image processing and tractography analyses (S. M. Smith et al., 2004; Woolrich et al., 2009). The T1 and DTI data were manually checked for motion and artifact. Registration was completed using (linear) FLIRT. Eddy current correction was performed to correct for induced distortions on the images from eddy currents in the gradient coils. DTFIT fit a diffusion tensor model at each voxel. The BEDPOST tool estimated distributions on the diffusion parameters for each voxel by running Markov Chain Monte Carlo sampling. Probabilistic tractography was carried out in native space with Probtrackx using regions of interest to seed the tract and compute the likely principal tract between a priori areas of the brain. The Probtrackx algorithm repetitively sampled from the distributions on voxel-wise principal diffusion directions to compute the streamline/tract (Behrens et al., 2003). This creates a distribution of the most likely dominant pathway between regions while taking into account uncertainty or error within each voxel (Behrens, Berg, Jbabdi, 
Rushworth, \& Woolrich, 2007). The output is a probability map of the connections between ROIs, which was then thresholded at 50\% and converted to standard MNI space. The tract was then used as an ROI and overlaid on the individual's FA map in MNI space and the average FA value was extracted from within the tract ROI. All tracts were visually inspected to ensure that the program accurately identified the expected and neuroanatomically consistent tract between regions of interest.

Regions of Interest for tract definition. Waypoint and exclusion masks were utilized to optimize the tract output. The inferior frontal and parietotemporal reading regions were hypothesized to be structurally connected through the arcuate fasciculus (sometimes considered part of the superior longitudinal fasciculus). Recent work on the structure of this tract has discovered that the arcuate fasciculus actually contains multiple tracts (Catani, Jones, \& ffytche, 2005): (1) connecting superior temporal with inferior frontal regions, (2) connecting inferior frontal gyrus (IFG) with the inferior parietal lobule (PT), (3) connecting superior temporal to the inferior parietal lobule (Catani \& Mesulam, 2008; Wakana et al., 2007). The specific tract that was chosen for this study was segment (2) connecting the IFG and PT areas. Published guidelines were used to create the ROIs to enter in the probabilistic tractography analyses to obtain the arcuate fasciculus (Catani \& Mesulam, 2008; Wakana, et al., 2007). Although a specific segment of the arcuate fasciculus was measured, this segment will be referred to as the arcuate fasciculus for this report (see Figure 1).

The structural connection between the IFG and the occipitotemporal area (OT) was hypothesized to be consisting of the inferior fronto-occipital fasciculus (IFOF) (Vandermosten et al., 2012). The IFOF runs anterior-posterior and connects visual association areas with frontal association areas. Previously published ROI guidelines were used to create the ROIs to enter in 
the probabilistic tractography analyses (see Figure 1) (Vandermosten, et al., 2012; Wakana, et al., 2007).

The specific tract connecting PT and OT reading areas was unknown and not well defined by a major tract. Therefore, regions of interest included gray matter areas in the PT and OT. The PT was defined as the Angular gyrus using the JHU Histological Atlas probabilistic map thresholded at 50\%. The OT area, more specifically, the Visual Word Form Area, is described as centering over the occipitotemporal sulcus but does not have specific gyral boundaries and has primarily been defined functionally. Therefore, the center coordinates of this area (MNI coordinates $\mathrm{x}:-44, \mathrm{y}:-58, \mathrm{z}:-15)$ were used as the center of a $5 \mathrm{~mm}$-radius sphere (Noble, Wolmetz, Ochs, Farah, \& McCandliss, 2006). This sphere was used as the ROI for the OT area as has been implemented in previous research (Noble, et al., 2006). The pathway connecting these areas likely runs in the superior-inferior direction (see Figure 1).

The cortico-spinal tract (CST) was chosen as a control tract given that it was not expected to be related to the neuroanatomically-defined reading system nor reading skills. Rather this tract is known to be associated with the relay of sensorimotor information (Binkofski et al., 1996; Schaechter, Perdue, \& Wang, 2008). Previously published ROI guidelines were used to create two ROIs to enter in the probabilistic tractography analyses (Wakana, et al., 2007).

Tracts were removed from analyses if the output included the incorrect tract or if the output did not identify a tract between the ROIs. Three participant's data ( 2 control, 1 survivor) did not produce a tract output for the PT-OT connection. Another three participant's data (2 control, 1 survivor) produced the incorrect tract from the ROIs intended to produce the IFOF. For one participant, the superior ROI for the CST was not able to be drawn due to tumor location in that area. These data could not be corrected and therefore were excluded from analyses. 
However, these participant's other tract data were appropriate and were used in analyses. With these specific tracts excluded, the sample size for each comparison was different. However, the sample size was never lower than 17 (control group) compared with 16 (survivor group).

\section{Procedure}

Cognitive measures were administered during one study visit while DTI scans occurred during a second study visit. With the exception of 3 individuals, the average time between testing and scanning was 1.94 months. Three participants had greater than 1 year between visits (17-67 months, 1 survivor, 2 control). We acknowledge that greater time between the cognitive data and DTI scans could be a confound; therefore we re-ran the correlation analyses excluding these 3 participants. The direction and strength of the relationships did not significantly change when these participants were excluded, therefore these participants were kept in the final analyses. Demographic variables were collected via self-report. Medical variables for survivors were collected through medical records. Participants were compensated for their time and travel.

\section{Analysis}

Demographic variables of age, sex, SES, and education were evaluated as potential confounds to be used as covariates in analyses. A confound was defined as a variable that was significantly related to the independent variable as well as correlated with the dependent variable. Education met these criteria for the IFOF tract only, thus education was entered as a covariate in analyses pertaining to the IFOF tract. For the AF and PT-OT tracts, the impact of education on the variance explained in the mediation models was investigated and suggested that education did not explain significant variance in word reading. Thus, due to the limited sample size, education was not included as a covariate in analyses with the AF and PT-OT. No other demographic variables met criteria for a confound (see Table 1). 
Correlation Analyses. To address the first aim of the study, to explore the relationship between reading skill and white matter integrity associated with the tracts connecting the three primary functional reading brain regions, correlations were conducted for the survivor and healthy control groups separately. Given that fractional anisotropy (FA) was expected to be positively related to word reading skill for both groups as seen in previous research (Vandermosten, et al., 2012), one-tailed correlations were run. We did not expect an association in the opposite direction. The following measures were correlated with WJ-III Letter Word Identification z- scores: FA in each of the three white matter connections using a partial correlation for IFOF and bivariate correlations for Arcuate fasciculus, and PT-OT. A p-value of less than .05 was considered a statistically significant relationship. Effect sizes (r values) were also evaluated.

Moderated Mediation Analyses. Guidelines developed by Preacher et al. (2007) were followed to investigate the role of processing speed as a mediator in the relationship between integrity of the white matter connection and reading skill with group membership as a moderator of the indirect effect. Model 5 (Preacher, et al., 2007) was utilized because Group was predicted to moderate both the $\mathrm{a}$ and $\mathrm{b}$ paths of the mediation model, thereby moderating the entire indirect effect.

The Dr. Andrew Hayes’ SPSS “modmed” macro was used (http://www.afhayes.com/spss-sas-and-mplus-macros-and-code.html). The model first calculates significance based on the Sobel test for the conditional indirect effects and provides $p$-values for significance levels. Then the model provides a 95\% bootstrap confidence interval (bias-corrected and accelerated) for each level of the moderator (with a dichotomous moderator). The number of bootstrap samples was set at 5,000. The bootstrap approach does not make assumptions about the 
shape of the sampling distribution like normal theory tests (Preacher, et al., 2007). Instead, through bootstrapping, the sampling distribution of the conditional main effect is estimated nonparametrically by sampling with replacement and the bootstrap sampling distribution is used to generate confidence intervals for the conditional indirect effect (Preacher, et al., 2007). Normal theory tests generated in the SPSS macro were verified by bootstrapping (Preacher, et al., 2007). Bootstrap confidence intervals (bias corrected and accelerated) for each level of the moderator informed the statistical significance of the conditional indirect effect in each group. A confidence interval that does not include zero was considered a statistically significant conditional indirect effect. The B value indicates the direction of the relationship, therefore a positive B value indicates that the survivors (group 1) show a greater mediating relationship than the control group (group 0). Group mediation effects were also compared using $\mathrm{R}^{2}$ values.

Control Tract and Task. To increase confidence that any findings in this study are related to reading skill specifically, and not an effect on general abilities, we investigated the relation between white matter integrity and skilled motor speed, as mediated by processing speed. The outcome variable was Grooved Pegboard dominant hand z-scores. The moderating variable was Group (Survivor=1, Control=0).

To be more confident that any findings in this study were related to the specific white matter areas studied, and not an effect on global white matter structures, we investigated the relation between white matter integrity (FA) in the cortico-spinal tract and reading achievement as mediated by processing speed.

\section{Results}

Descriptive statistics (see Table 1) demonstrate that the groups differed on measures of FA of the Arcuate Fasciculus (AF), word reading, and skilled motor speed. Even so, reading 
abilities appeared to be mostly intact in this group of survivors, with $82 \%$ of survivors exhibiting scores at or above 1.5 SD below mean. Obtaining correlations by excluding the impaired scores $(n=3)$ from the survivor group did not change the results. Therefore, we included them in all analyses. In contrast to previous research on IQ changes in childhood brain tumor survivors, IQ measures did not differentiate the groups (Palmer, 2008).

\section{Aim 1}

Aim 1 examined the relationship between word reading scores and the three hypothesized reading-related white matter tracts (see Figure 2). For the IFOF, both groups showed a significant association with reading (survivor: $\mathrm{r}(14)=.45, \mathrm{p} \leq .05$; control: $\mathrm{r}(14)=.58, \mathrm{p}=.009$ ). Thus, higher white matter integrity was associated with better word reading scores. For the AF, white matter integrity did not show a significant relationship with reading scores in either group and the effect sizes were small (survivor: $r(17)=.05, \mathrm{p}>.05$; control: $\mathrm{r}(18)=-.03, \mathrm{p}>.05$ ). The white matter integrity of the PT-OT was significantly correlated with reading for the survivor group $(\mathrm{r}(16)=.46, \mathrm{p} \leq .03)$ but not for the control group. However, the control group evidenced a medium effect size $(\mathrm{r}(16)=.40, \mathrm{p} \leq .06)$. In sum, the white matter microstructure of the tracts extending from the OT area (IFOF and PT-OT) show medium to large correlations with word reading.

\section{Aim 2}

Aim 2 hypothesized that processing speed would mediate the relationship between white matter integrity of each tract studied and word reading, and this mediation would be moderated by group. In the moderated mediation analysis, the mediator variable model indicated that the interaction between group and IFOF was significant (see Figure $3 ; \mathrm{b}=28.20, \mathrm{t}=2.12, \mathrm{p}<.05$ ). The dependent variable model indicated that the interaction between group and processing speed was 
significant $(b=.59, t=2.60, p<.05)$. Thus, group membership significantly moderated $a$ and $b$ paths of the model such that the survivor group (Group 1) evidenced a stronger indirect effect. The moderation of the conditional indirect effect was verified with bootstrapping with 5,000 samples. The $95 \%$ bootstrap bias corrected accelerated confidence interval did not contain zero for the survivor group confirming the statistical significance of the conditional indirect effect in this group. Further, the variance explained by the mediation model in the survivor group was $61 \%$ and $45 \%$ in the control group. The result of this moderated mediation is consistent with our prediction for Aim 2.

With the Arcuate Fasciculus (AF) entered as the independent variable, the effect of the interaction between group and AF integrity was not significant which was confirmed by bootstrapping. Thus, this moderated mediation model is not supported with AF as the predictor (see Figure 3).

When the PT-OT tract was tested as the predictor variable in the moderated mediation analysis, the mediator variable model indicated that the interaction between group and PT-OT was significant (see Figure 3; $\mathrm{b}=21.27, \mathrm{t}=2.08, \mathrm{p}<.05$ ). The dependent variable model indicated that the interaction between group and processing speed was significant $(b=.66, t=2.97, p<.01)$. Thus, group membership significantly moderated a and b paths of the model such that the survivor group (Group 1) evidenced a stronger indirect effect. The moderation of the conditional indirect effect was verified with bootstrapping with 5,000 samples. The $95 \%$ bootstrap bias corrected accelerated confidence interval did not contain zero for the survivor group confirming the statistical significance of the conditional indirect effect in this group. Further, the variance explained by the mediation model in the survivor group was $61 \%$ and $18 \%$ in the control group. The result of this moderated mediation is consistent with our prediction for Aim 2. 


\section{Model Specificity}

When the moderated mediation model was tested with the corticospinal tract (CST), the interaction between CST integrity and group did not significantly predict processing speed $(b=10.70, S E=9.33, p>.05)$. Bootstrapping confirmed the lack of significance.

Skilled motor speed was entered in the moderated mediation model, with separate models for each reading tract. For IFOF, the correlation with skilled motor speed indicated a significant positive relationship $(\mathrm{r}(16)=.52, \mathrm{p}<.05)$. However, in accordance with predictions, the moderated mediation models were not significant with either of the three reading tracts. Thus, for skilled motor speed that was not hypothesized to be related to reading skill, the moderated mediation model was not significant with tracts in the reading system.

\section{Discussion}

Building upon an existing neurodevelopmental models of reading, processing speed was found to improve the prediction of the relationship between white matter microstructure and word reading for two of three tracts studied. White matter integrity was related to reading scores but this association was mediated by processing speed. Group significantly moderated the conditional indirect effect of white matter on word reading, with the mediation model being significant for the survivor group but not for the control group.

\section{Moderated Mediation Model}

The significant moderated mediation, indicating a conditional indirect effect of group, supports and builds upon the neurodevelopmental model of outcomes in childhood brain tumors (Palmer, 2008). The current study's model of the relationships between white matter, reading and processing speed is in line with our hypotheses and consistent with previous work studying the 
relationship among these constructs. Poorer reading has been shown to be related to poorer processing speed in individuals with brain injury and with reading disabilities (Barnes, Dennis, \& Wilkinson, 1999; Shanahan, et al., 2006), and our study demonstrates that this relationship extends to childhood brain tumor survivors. Analyses with control tract and control task suggest the moderated mediation model is specific to word reading and the IFOF and PT-OT tracts rather than broader cognitive outcomes or global white matter microstructure, which strengthens the specificity of the model.

For both the PT-OT and the IFOF, group membership (survivor vs controls) was found to moderate the indirect effect of white matter integrity on word reading through processing speed. The findings supported the hypothesis and indicated that in the survivor group, individual differences in processing speed improved the prediction of the relationship between white matter integrity and word reading. In the control group, this relationship was not predicted and indeed, processing speed was not a mediator. Our moderated mediation analysis provided statistical evidence that the indirect effect of processing speed was dependent on group membership. Our model illustrates that how white matter integrity and reading are related is a critical difference between the groups. This model lends support for the developmental cascade model as demonstrated by the effect of processing speed in survivors despite similar means between the groups. The developmental cascade hypothesis predicts that deficits in core cognitive skills such as processing speed in childhood cascade over time to adversely affect the learning and development of cognitive outcomes (Fry \& Hale, 1996, 2000; Palmer, 2008). This is consistent with empirical evidence that brain tumor survivors experience a slower rate of learning compared to peers. 
The development of core cognitive skills, particularly processing speed in this study, may be crucial in translating structural connectivity into reading skill for survivors. Reading is a complex learned skill, in which brain areas for language, speech, and vision processing must be integrated to achieve effective phonological processing, word analysis, fluency, and comprehension. Therefore, it is reasonable that core cognitive skills may serve as developmental scaffolding as reading is learned and practiced.

\section{White Matter Integrity and Word Reading}

We found significant associations between the white matter integrity of the IFOF and PTOT connection and word reading skill for childhood brain tumor survivors and typically developing individuals suggesting these pathways are important for word reading in adults regardless of history of brain tumor. These findings are in line with previous research that purports a similar relationship between white matter integrity and reading for both skilled and poor readers irrespective of prior childhood brain tumor (Niogi \& McCandliss, 2006). The current study replicates IFOF findings from a recent study using a similar ROI method in adults, and adds the PT-OT as an important pathway particularly in brain tumor survivors (Vandermosten, et al., 2012). With regard to the arcuate fasciculus, recent work suggests that AF may be more specifically focused on phonological awareness aspects of reading, which may explain the current study's lack of findings with the AF (Vandermosten, et al., 2012).

\section{Importance of Occipitotemporal Pathways}

The current study compliments previous research by demonstrating that tracts from the OT area to reading regions defined by functional MRI (fMRI) are critical for reading in adult survivors and controls. Prior research used exploratory or whole-brain methods such as Tract Based Spatial Statistics and Voxel Based Analysis, which has led to some confusion when the 
location and orientation of a key finding in the parietotemporal area did not correspond to extant knowledge about connections between cortical areas. This has led researchers to question how white matter findings correspond with the current fMRI model of reading. Although whole brain analysis methods can be fruitful in discovering unexpected yet meaningful correlations, it is also important to examine relationships using empirically driven and a priori theoretically driven models (Niogi \& McCandliss, 2006).

The results suggest that the OT area is a critical junction in the reading system for young adults. This area has been shown to play an important role in fluid reading as reading skill progresses. Differences in the function of this region between poor and skilled readers has led researchers to suggest the functional breakdown in the OT is a biomarker for reading disabilities (Pugh et al., 2000). The white matter from the OT connecting with the inferior frontal gyrus (IFG) and PT areas as measured by the IFOF and PT-OT tracts in this study may underlie these findings in functional MRI studies of reading. Together these findings emphasize the importance of continued examination of white matter pathways emanating from the OT.

The association of PT-OT tract to word reading is not surprising, given that damage to the angular gyrus and its associated connections with the occipitotemporal area results in alexia in which one is unable to read (Damasio \& Damasio, 1983). Past research also has found a functional disconnection between the angular gyrus and occipitotemporal regions in adult males with dyslexia (Horwitz, Rumsey, \& Donohue, 1998). In children, typical readers showed functional connectivity between the OT and IFG, whereas poor readers seemed to rely on a different connection and showed functional connectivity between the OT and right prefrontal areas (S. E. Shaywitz et al., 2003). Furthermore, a recent resting state functional connectivity study found that reading was positively associated with functional connectivity from the OT area 
to both the IFG and PT (inferior parietal lobule) in adults (Koyama et al., 2011). However, functional connectivity is a measure of the temporal correlation between separate regions of activation and does not necessarily imply structural connectivity (Fingelkurts, Fingelkurts, \& Kahkonen, 2005). This study adds to the increasing evidence for role of OT in reading development and helps build a richer theoretical model of reading in which functional and structural connectivity findings complement each other. Along these lines, future work could structurally examine the different functional connections used by poor readers described earlier (S. E. Shaywitz, et al., 2003).

\section{Word reading in the current sample of survivors}

Although only $18 \%$ of the survivors in our sample ( 3 individuals) had word reading performance in the clinically impaired range (1.5 or more standard deviations below the mean), their scores on average were significantly lower than controls. This finding is in line with previous research showing greater risk for reading difficulties in survivors (Robinson, et al., 2010). The low proportion of impaired word reading performance might be explained by the fact that Letter-Word ID is an untimed task and therefore may underrepresent the real world reading difficulties experienced by survivors in the classroom or a work setting.

Due to the larger sample needed to detect a significant effect these findings should be considered preliminary until replication with a larger sample (Fritz \& Mackinnon, 2007). A limitation of this study was the necessary exclusion criteria of individuals with metal artifact or large anatomical abnormalities. The current sample of survivors may represent a "best outcome" group and findings may not generalize to survivors with greater neurological complications. That said, $44 \%$ of our survivors had radiation and chemotherapy as treatment (8/18), and $33 \%$ had hydrocephalus. In addition, the PT-OT pathway is less well defined by a major neuroanatomical 
tract. However, empirical research on the fMRI-based neural reading circuit guided the choice of regions of interest. Different ROI methods were utilized for the PT-OT connection compared to the other connections and this must be taken into account when comparing results. More work is needed to determine the effect of disease (e.g. tumor type and location), treatment factors (e.g. cranial radiation therapy, chemotherapy, hydrocephalus, seizures), age at diagnosis, and time since diagnosis for long-term childhood brain tumor survivors. Exploratory analyses in the current sample suggested that age at diagnosis explained $9 \%$ additional unique variance in the survivor mediation model; radiation/chemotherapy explained $2 \%$ additional variance. However a larger sample is needed to address the complexities of these treatment factors on outcomes in adult survivors.

A strength of this study was the use of the empirical literature to drive predictions about specific tracts. The chosen tracts (IFOF and AF) and regions of interest (PT-OT) were based on the extensive literature on the functional MRI based reading system and the hypothesized mechanisms of dysfunction that occur in individuals with dyslexia (i.e. poor communication between reading areas). Comparing the tracts within an empirically-based system can allow researchers to test theoretical relationships and develop models to investigate potential breakdown in the system. However, a structural connectome or network based approach would allow for examination of network interconnections rather than as separate tracts hypothesized to connect nodes. A connectome approach is based on the motivation that brain function is a result of the topology of complex networks rather than individual regions or tracts alone (van den Heuvel \& Sporns, 2011). Future research on the reading network using a connectome approach would complement the current study. 
Given that in the current study, the relationships between specific white matter tract integrity, processing speed, and reading are present in early adulthood survivors, the most advantageous interventions may be ones that strengthen the link between processing speed and reading. Such interventions may focus on the automaticity and speed of reading to help prevent slow processing speed from cascading into poorer reading outcomes. Currently, reading interventions are being developed and tested that focus on dual aspects of reading difficulties: speed and decoding (Katzir et al., 2006; Wolf, Miller, \& Donnelly, 2000).

\section{Conclusions}

The current study integrated the neuroimaging-derived reading neural system literature and the childhood brain tumor literature to strengthen and develop a richer neurodevelopmental model for long-term brain tumor survivors. The findings support the model that white matter pathways emanating from the occipitotemporal area are important for word reading in adults. In addition, in brain tumor survivors, processing speed is a mechanism by which the relationship between white matter integrity and word reading is impacted. The research on white matter integrity of pathways underlying functional neural systems is growing as innovative neuroimaging technology and analysis approaches rapidly accelerate. Examining the underlying white matter structure is an important and major force in piecing together the development of typical and disrupted reading skill. 


\section{References}

Assaf, Y., \& Pasternak, O. (2008). Diffusion tensor imaging (DTI)-based white matter mapping in brain research: a review. J Mol Neurosci, 34(1), 51-61. doi: 10.1007/s12031-007-00290

Barnes, M., Dennis, M., \& Wilkinson, M. (1999). Reading after closed head injury in childhood: effects on accuracy, fluency, and comprehension. Developmental Neuropsychology 15(1), 1-24.

Beaulieu, C., Plewes, C., Paulson, L. A., Roy, D., Snook, L., Concha, L., \& Phillips, L. (2005). Imaging brain connectivity in children with diverse reading ability. Neuroimage, 25(4), 1266-1271. doi: S1053-8119(04)00798-0

Beebe, D. W., Ris, M. D., Armstrong, F. D., Fontanesi, J., Mulhern, R., Holmes, E., \& Wisoff, J. H. (2005). Cognitive and adaptive outcome in low-grade pediatric cerebellar astrocytomas: evidence of diminished cognitive and adaptive functioning in National Collaborative Research Studies (CCG 9891/POG 9130). J Clin Oncol, 23(22), 51985204. doi: $23 / 22 / 5198$

Behrens, T. E., Berg, H. J., Jbabdi, S., Rushworth, M. F., \& Woolrich, M. W. (2007). Probabilistic diffusion tractography with multiple fibre orientations: What can we gain? Neuroimage, 34(1), 144-155. doi: 10.1016/j.neuroimage.2006.09.018

Behrens, T. E., Woolrich, M. W., Jenkinson, M., Johansen-Berg, H., Nunes, R. G., Clare, S., . . Smith, S. M. (2003). Characterization and propagation of uncertainty in diffusionweighted MR imaging. Magn Reson Med, 50(5), 1077-1088. doi: 10.1002/mrm.10609 
Binkofski, F., Seitz, R. J., Arnold, S., Classen, J., Benecke, R., \& Freund, H. J. (1996). Thalamic metbolism and corticospinal tract integrity determine motor recovery in stroke. Ann Neurol, 39(4), 460-470. doi: 10.1002/ana.410390408

Booth, J. R., Burman, D. D., Van Santen, F. W., Harasaki, Y., Gitelman, D. R., Parrish, T. B., \& Marsel Mesulam, M. M. (2001). The development of specialized brain systems in reading and oral-language. Child Neuropsychol, 7(3), 119-141.

Bornstein, R. A. (1985). Normative data on selected neuropsychological measures from a nonclinical sample. Journal of Clinical Psychology, 41(5), 651-659.

Briere, M. E., Scott, J. G., McNall-Knapp, R. Y., \& Adams, R. L. (2008). Cognitive outcome in pediatric brain tumor survivors: delayed attention deficit at long-term follow-up. Pediatr Blood Cancer, 50(2), 337-340. doi: 10.1002/pbc.21223

Cascio, C. J., Gerig, G., \& Piven, J. (2007). Diffusion tensor imaging: Application to the study of the developing brain. J Am Acad Child Adolesc Psychiatry, 46(2), 213-223. doi: 10.1097/01.chi.0000246064.93200.e8S0890-8567(09)61829-3

Catani, M., Jones, D. K., \& ffytche, D. H. (2005). Perisylvian language networks of the human brain. Annals of neurology, 57(1), 8-16. doi: 10.1002/ana.20319

Catani, M., \& Mesulam, M. (2008). The arcuate fasciculus and the disconnection theme in language and aphasia: history and current state. Cortex, 44(8), 953-961. doi: 10.1016/j.cortex.2008.04.002

Catts, H. W., Gillispie, M., Leonard, L. B., Kail, R. V., \& Miller, C. A. (2002). The role of speed of processing, rapid naming, and phonological awareness in reading achievement. $J$ Learn Disabil, 35(6), 509-524. 
Christodoulou, C., Krupp, L. B., Liang, Z., Huang, W., Melville, P., Roque, C., . . Peyster, R. (2003). Cognitive performance and MR markers of cerebral injury in cognitively impaired MS patients. Neurology, 60(11), 1793-1798.

Conklin, H. M., Li, C., Xiong, X., Ogg, R. J., \& Merchant, T. E. (2008). Predicting change in academic abilities after conformal radiation therapy for localized ependymoma. J Clin Oncol, 26(24), 3965-3970. doi: 26/24/3965 10.1200/JCO.2007.15.9970

Damasio, A. R., \& Damasio, H. (1983). The anatomic basis of pure alexia. Neurology, 33(12), $1573-1583$.

Deutsch, G. K., Dougherty, R. F., Bammer, R., Siok, W. T., Gabrieli, J. D., \& Wandell, B. (2005). Children's reading performance is correlated with white matter structure measured by diffusion tensor imaging. Cortex, 41(3), 354-363.

Fingelkurts, A. A., Fingelkurts, A. A., \& Kahkonen, S. (2005). Functional connectivity in the brain--is it an elusive concept? Neurosci Biobehav Rev, 28(8), 827-836. doi: 10.1016/j.neubiorev.2004.10.009

First, M. B., Spitzer, R. L., Gibbon, M., \& Williams, J. B. W. (2002). Structured Clinical Interview for DSM-IV-TR Axis I Disorders, Research Version, Patient Edition. (SCIDI/P). New York: Biometrics Research, New York State Psychiatric Institute.

Fritz, M. S., \& Mackinnon, D. P. (2007). Required sample size to detect the mediated effect. Psychological science, 18(3), 233-239. doi: 10.1111/j.1467-9280.2007.01882.x

Fry, A. F., \& Hale, S. (1996). Processing speed, working memory, and fluid intelligence: evidence for a developmental cascade. Psychological Science, 7, 237-241.

Fry, A. F., \& Hale, S. (2001). Relationships among processing speed, working memory, and fluid intelligence in children. Biol Psychol, 54(1-3), 1-34. doi: S030105110000051X 
Frye, R. E., Hasan, K., Xue, L., Strickland, D., Malmberg, B., Liederman, J., \& Papanicolaou, A. (2008). Splenium microstructure is related to two dimensions of reading skill. Neuroreport, 19(16), 1627-1631. doi: 10.1097/WNR.0b013e328314b8ee

Frye, R. E., Liederman, J., Hasan, K. M., Lincoln, A., Malmberg, B., McLean, J., 3rd, \& Papanicolaou, A. (2010). Diffusion tensor quantification of the relations between microstructural and macrostructural indices of white matter and reading. Hum Brain Mapp, 32, 1220-1235 doi: 10.1002/hbm.21103

Glauser, T. A., \& Packer, R. J. (1991). Cognitive deficits in long-term survivors of childhood brain tumors. Childs Nerv Syst, 7(1), 2-12.

Hollingshead, A. B. (1975). Four Factor Index of Social Status. Department of Sociology. Yale University.

Horwitz, B., Rumsey, J. M., \& Donohue, B. C. (1998). Functional connectivity of the angular gyrus in normal reading and dyslexia. Proc Natl Acad Sci U S A, 95(15), 8939-8944.

Katzir, T., Kim, Y., Wolf, M., O'Brien, B., Kennedy, B., Lovett, M., \& Morris, R. (2006). Reading fluency: the whole is more than the parts. Ann Dyslexia, 56(1), 51-82. doi: $10.1007 / \mathrm{s} 11881-006-0003-5$

Kieffer-Renaux, V., Bulteau, C., Grill, J., Kalifa, C., Viguier, D., \& Jambaque, I. (2000). Patterns of neuropsychological deficits in children with medulloblastoma according to craniospatial irradiation doses. Dev Med Child Neurol, 42(11), 741-745.

Klingberg, T., Hedehus, M., Temple, E., Salz, T., Gabrieli, J. D., Moseley, M. E., \& Poldrack, R. A. (2000). Microstructure of temporo-parietal white matter as a basis for reading ability: evidence from diffusion tensor magnetic resonance imaging. Neuron, 25(2), 493-500. doi: S0896-6273(00)80911-3 
Koyama, M. S., Di Martino, A., Zuo, X. N., Kelly, C., Mennes, M., Jutagir, D. R., .. . Milham, M. P. (2011). Resting-state functional connectivity indexes reading competence in children and adults. The Journal of neuroscience : the official journal of the Society for Neuroscience, 31(23), 8617-8624. doi: 10.1523/JNEUROSCI.4865-10.2011

Mabbott, D. J., Penkman, L., Witol, A., Strother, D., \& Bouffet, E. (2008). Core neurocognitive functions in children treated for posterior fossa tumors. Neuropsychology, 22(2), 159168. doi: 2008-02526-003

Mabbott, D. J., Spiegler, B. J., Greenberg, M. L., Rutka, J. T., Hyder, D. J., \& Bouffet, E. (2005). Serial evaluation of academic and behavioral outcome after treatment with cranial radiation in childhood. J Clin Oncol, 23(10), 2256-2263. doi: 23/10/2256

Mori, S., \& van Zijl, P. C. (2002). Fiber tracking: principles and strategies - a technical review. NMR Biomed, 15(7-8), 468-480. doi: 10.1002/nbm.781

Mulhern, R. K., \& Palmer, S. L. (2003). Neurocognitive late effects in pediatric cancer. Curr Probl Cancer, 27(4), 177-197. doi: S0147027203000266

Mulhern, R. K., Palmer, S. L., Merchant, T. E., Wallace, D., Kocak, M., Brouwers, P., .. . Gajjar, A. (2005). Neurocognitive consequences of risk-adapted therapy for childhood medulloblastoma. J Clin Oncol, 23(24), 5511-5519. doi: 23/24/5511

Niogi, S. N., \& McCandliss, B. D. (2006). Left lateralized white matter microstructure accounts for individual differences in reading ability and disability. Neuropsychologia, 44(11), 2178-2188. doi: S0028-3932(06)00023-6

Noble, K. G., Wolmetz, M. E., Ochs, L. G., Farah, M. J., \& McCandliss, B. D. (2006). Brainbehavior relationships in reading acquisition are modulated by socioeconomic factors. Dev Sci, 9(6), 642-654. doi: 10.1111/j.1467-7687.2006.00542.x 
Odegard, T. N., Farris, E. A., Ring, J., McColl, R., \& Black, J. (2009). Brain connectivity in nonreading impaired children and children diagnosed with developmental dyslexia. Neuropsychologia, 47(8-9), 1972-1977. doi: S0028-3932(09)00124-9

Palmer, S. L. (2008). Neurodevelopmental impact on children treated for medulloblastoma: a review and proposed conceptual model. Dev Disabil Res Rev, 14(3), 203-210. doi: 10.1002/ddrr.32

Palmer, S. L., Goloubeva, O., Reddick, W. E., Glass, J. O., Gajjar, A., Kun, L., .. Mulhern, R. K. (2001). Patterns of intellectual development among survivors of pediatric medulloblastoma: a longitudinal analysis. J Clin Oncol, 19(8), 2302-2308.

Palmer, S. L., Reddick, W. E., Glass, J. O., Ogg, R., Patay, Z., Wallace, D., \& Gajjar, A. (2010). Regional white matter anisotropy and reading ability in patients treated for pediatric embryonal tumors. Brain Imaging Behav, 4(2), 132-140. doi: 10.1007/s11682-010-90921

Parmenter, B. A., Weinstock-Guttman, B., Garg, N., Munschauer, F., \& Benedict, R. H. (2007). Screening for cognitive impairment in multiple sclerosis using the Symbol digit Modalities Test. Mult Scler, 13(1), 52-57.

Perrine, K., Hermann, B. P., Meador, K. J., Vickrey, B. G., Cramer, J. A., Hays, R. D., \& Devinsky, O. (1995). The relationship of neuropsychological functioning to quality of life in epilepsy. Arch Neurol, 52(10), 997-1003.

Preacher, K. J., Rucker, D. D., \& Hayes, A. F. (2007). Addressing moderated mediation hypotheses: theory, methods, and prescriptions. Multivariate behavioral research, 42(1), 185-227. 
Pugh, K. R., Frost, S. J., Sandak, R., Landi, N., Moore, D., Della Porta, G., . . Mencl, E. (2010). Mapping the word reading circuitry in skilled and disabled readers. In P. L. Cornelissen, P. C. Hansen, M. L. Kringelbach \& K. R. Pugh (Eds.), The Neural Basis of Reading (pp.281-305). New York: Oxford University Press.

Pugh, K. R., Mencl, W. E., Jenner, A. R., Katz, L., Frost, S. J., Lee, J. R., . . Shaywitz, B. A. (2000). Functional neuroimaging studies of reading and reading disability (developmental dyslexia). Ment Retard Dev Disabil Res Rev, 6(3), 207-213. doi: 10.1002/10982779(2000)6:3<207::AID-MRDD8>3.0.CO;2-P

Pugh, K. R., Mencl, W. E., Jenner, A. R., Katz, L., Frost, S. J., Lee, J. R., . . Shaywitz, B. A. (2001). Neurobiological studies of reading and reading disability. J Commun Disord, 34(6), 479-492.

Reddick, W. E., White, H. A., Glass, J. O., Wheeler, G. C., Thompson, S. J., Gajjar, A., .. . Mulhern, R. K. (2003). Developmental model relating white matter volume to neurocognitive deficits in pediatric brain tumor survivors. Cancer, 97(10), 2512-2519. doi: $10.1002 /$ cncr.11355

Reeves, C. B., Palmer, S. L., Reddick, W. E., Merchant, T. E., Buchanan, G. M., Gajjar, A., \& Mulhern, R. K. (2006). Attention and memory functioning among pediatric patients with medulloblastoma. J Pediatr Psychol, 31(3), 272-280. doi: jsj019

Robinson, K. E., Kuttesch, J. F., Champion, J. E., Andreotti, C. F., Hipp, D. W., Bettis, A., .. . Compas, B. E. (2010). A quantitative meta-analysis of neurocognitive sequelae in survivors of pediatric brain tumors. Pediatr Blood Cancer, 55(3), 525-531. doi: $10.1002 /$ pbc. 22568 
Ruff, R. M., \& Parker, S. B. (1993). Gender- and age-specific changes in motor speed and eyehand coordination in adults: normative values for the Finger Tapping and Grooved Pegboard Tests. Percept Mot Skills, 76(3 Pt 2), 1219-1230.

Schaechter, J. D., Perdue, K. L., \& Wang, R. (2008). Structural damage to the corticospinal tract correlates with bilateral sensorimotor cortex reorganization in stroke patients. Neuroimage, 39(3), 1370-1382. doi: 10.1016/j.neuroimage.2007.09.071

Shanahan, M. A., Pennington, B. F., Yerys, B. E., Scott, A., Boada, R., Willcutt, E. G., . . . DeFries, J. C. (2006). Processing speed deficits in attention deficit/hyperactivity disorder and reading disability. J Abnorm Child Psychol, 34(5), 585-602. doi: 10.1007/s10802006-9037-8

Shaywitz, B. A., Shaywitz, S. E., Pugh, K. R., Mencl, W. E., Fulbright, R. K., Skudlarski, P., .. . Gore, J. C. (2002). Disruption of posterior brain systems for reading in children with developmental dyslexia. Biol Psychiatry, 52(2), 101-110. doi: S0006322302013653

Shaywitz, B. A., Skudlarski, P., Holahan, J. M., Marchione, K. E., Constable, R. T., Fulbright, R. K., . . Shaywitz, S. E. (2007). Age-related changes in reading systems of dyslexic children. Ann Neurol, 61(4), 363-370. doi: 10.1002/ana.21093

Shaywitz, S. E., \& Shaywitz, B. A. (2008). Paying attention to reading: the neurobiology of reading and dyslexia. Dev Psychopathol, 20(4), 1329-1349. doi: S0954579408000631

Shaywitz, S. E., Shaywitz, B. A., Fulbright, R. K., Skudlarski, P., Mencl, W. E., Constable, R. T., ... Gore, J. C. (2003). Neural systems for compensation and persistence: young adult outcome of childhood reading disability. Biol Psychiatry, 54(1), 25-33. doi: S000632230201836X 
Smith, A. (1982). Symbol digit modalities test manual, revised Los Angeles: Western Psychological Services.

Smith, S. M., Jenkinson, M., Woolrich, M. W., Beckmann, C. F., Behrens, T. E., Johansen-Berg, H., . . Matthews, P. M. (2004). Advances in functional and structural MR image analysis and implementation as FSL. Neuroimage, 23 Suppl 1, S208-219. doi: S10538119(04)00393-3

Tournier, J. D., Mori, S., \& Leemans, A. (2011). Diffusion tensor imaging and beyond. Magn Reson Med, 65(6), 1532-1556. doi: 10.1002/mrm.22924

van den Heuvel, M. P., \& Sporns, O. (2011). Rich-club organization of the human connectome. The Journal of neuroscience : the official journal of the Society for Neuroscience, 31(44), 15775-15786. doi: 10.1523/JNEUROSCI.3539-11.2011

Vandermosten, M., Boets, B., Poelmans, H., Sunaert, S., Wouters, J., \& Ghesquiere, P. (2012). A tractography study in dyslexia: neuroanatomic correlates of orthographic, phonological and speech processing. Brain, 135(Pt 3), 935-948. doi: 10.1093/brain/awr363

Wakana, S., Caprihan, A., Panzenboeck, M. M., Fallon, J. H., Perry, M., Gollub, R. L., ... Mori, S. (2007). Reproducibility of quantitative tractography methods applied to cerebral white matter. Neuroimage, 36(3), 630-644. doi: 10.1016/j.neuroimage.2007.02.049

Wechsler, D. (1999). WASI Manual. San Antonio: Psychological Corporation.

Wheeler-Kingshott, C. A., \& Cercignani, M. (2009). About "axial" and "radial" diffusivities. Magn Reson Med, 61(5), 1255-1260. doi: 10.1002/mrm.21965

Wolf, M., Miller, L., \& Donnelly, K. (2000). Retrieval, automaticity, vocabulary elaboration, orthography (RAVE-O): a comprehensive, fluency-based reading intervention program. $J$ Learn Disabil, 33(4), 375-386. 
Woodcock, R. W., McGrew, K. S., \& Mather, N. (2001a). Examiner's manual: Woodcock Johnson III Tests of Achievement. Itasca: Riverside.

Woodcock, R. W., McGrew, K. S., \& Mather, N. (2001b). Woodcock-Johnson (Third ed.). Itasca: Riverside.

Woolrich, M. W., Jbabdi, S., Patenaude, B., Chappell, M., Makni, S., Behrens, T., . . Smith, S. M. (2009). Bayesian analysis of neuroimaging data in FSL. Neuroimage, 45(1 Suppl), S173-186. doi: S1053-8119(08)01204-4 
Table 1

Demographics and descriptive statistics by group, correlations with word reading, and group differences

\begin{tabular}{|c|c|c|c|c|c|c|}
\hline & Survivor & Control & \multirow{2}{*}{\multicolumn{2}{|c|}{$\frac{\text { Group differences }}{\chi^{2} p \text { (2-tailed) }}$}} & \multicolumn{2}{|c|}{ Correlations } \\
\hline & \multicolumn{2}{|c|}{ Percent } & & & $\rho$ & $\mathrm{p}$ (2-tailed) \\
\hline Sex & $61 \% \mathrm{~F}$ & $63 \% \mathrm{~F}$ & 0.02 & NS & -0.09 & NS \\
\hline Ethnicity & $72 \%$ Caucasian & $50 \%$ Caucasian & 1.77 & NS & -0.07 & NS \\
\hline \multirow[t]{2}{*}{$\underline{\text { SES status }}$} & $88 \%$ High & $71 \%$ High & 1.41 & NS & 0.09 & NS \\
\hline & \multicolumn{2}{|c|}{ Mean (SD) } & $\mathrm{t}$ & $\mathrm{p}$ (2-tailed) & $\mathrm{r}$ & $\mathrm{p}$ (2-tailed) \\
\hline Age & $24.81(4.24)$ & $23.61(4.80)$ & -0.80 & NS & -0.02 & NS \\
\hline Education & $13.83(1.69)$ & $14.47(1.30)$ & 0.39 & NS & 0.41 & $<0.05$ \\
\hline $\begin{array}{l}\text { Inferior fronto-occipital } \\
\text { fasciculus (IFOF) }\end{array}$ & $0.35(0.03)$ & $0.36(0.02)$ & 1.38 & 0.18 & & \\
\hline Arcuate fasciculus (AF) * & $0.33(0.02)$ & $0.34(0.02)$ & 2.13 & 0.04 & & \\
\hline Parietotemporal - & $029\left(\begin{array}{l}0 \\
0\end{array}\right.$ & $031\left(\begin{array}{lll}0 & 03\end{array}\right)$ & 123 & 023 & & \\
\hline Cortico-spinal Tract (CST) & $0.44(0.04)$ & $0.45(0.05)$ & 0.43 & 0.67 & & \\
\hline $\begin{array}{l}\text { WJ- Letter-word identification } \\
\text { (LWID)* }\end{array}$ & $-0.37(0.90)$ & $0.36(0.55)$ & 2.98 & $<0.01$ & & \\
\hline Oral- Symbol-Digit Modalities & & & & & & \\
\hline Test (OSDMT) & $-0.69(1.24)$ & $-0.16(0.84)$ & 1.55 & 0.13 & & \\
\hline $\begin{array}{l}\text { Grooved Pegboard (Dominant } \\
\text { hand)* }\end{array}$ & $-1.31(1.02)$ & $-0.54(0.98)$ & 2.35 & 0.03 & & \\
\hline WASI- Performance IQ (PIQ) & $103.72(14.39)$ & $109.05(11.27)$ & 1.26 & 0.22 & & \\
\hline WASI- Verbal IQ (VIQ) & $97.78(19.28)$ & $106.68(11.39)$ & 1.70 & 0.10 & & \\
\hline WASI- Full scale (FSIQ) & $100.89(17.78)$ & $109.21(10.83)$ & 1.71 & 0.10 & & \\
\hline
\end{tabular}

Note. Pearson correlation coefficient was used for continuous variables: age and education.

Nonparametric correlations (Spearman's rho) was used for categorical variables of: sex, ethnicity, and socioeconomic status. Pearson Chi-Square test was used for sex while the Fisher Exact test was used for Ethnicity and Socioeconomic status (SES). All scores for neuropsychological measures are presented as $z$-scores except IQ presented as standard scores. All scores for white matter structures are presented as Fractional Anisotropy (FA) values. * indicates statistically significant group difference $(\mathrm{p}<.05)$. 


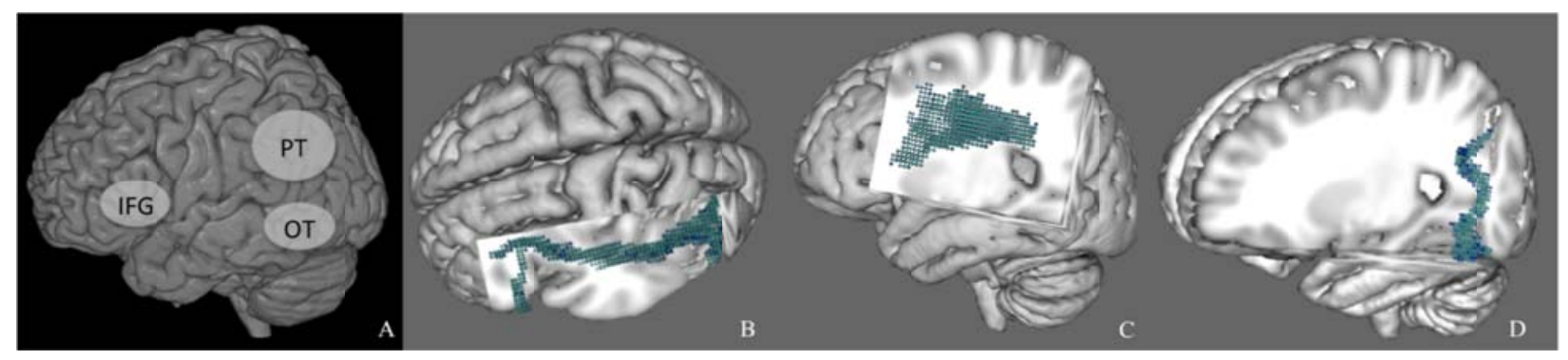

Figure 1. Model of fMRI-based reading regions (A) and example of individual tract outputs for pathways hypothesized to connect reading regions: IFOF (B), AF (C), PT-OT (D). Average tract fractional anisotropy (FA) used in analyses. IFG- inferior frontal gyrus, PT- parietotemporal area, OT- occipitotemporal area, IFOF- inferior fronto-occipital fasciculus, AF- arcuate fasciculus. 

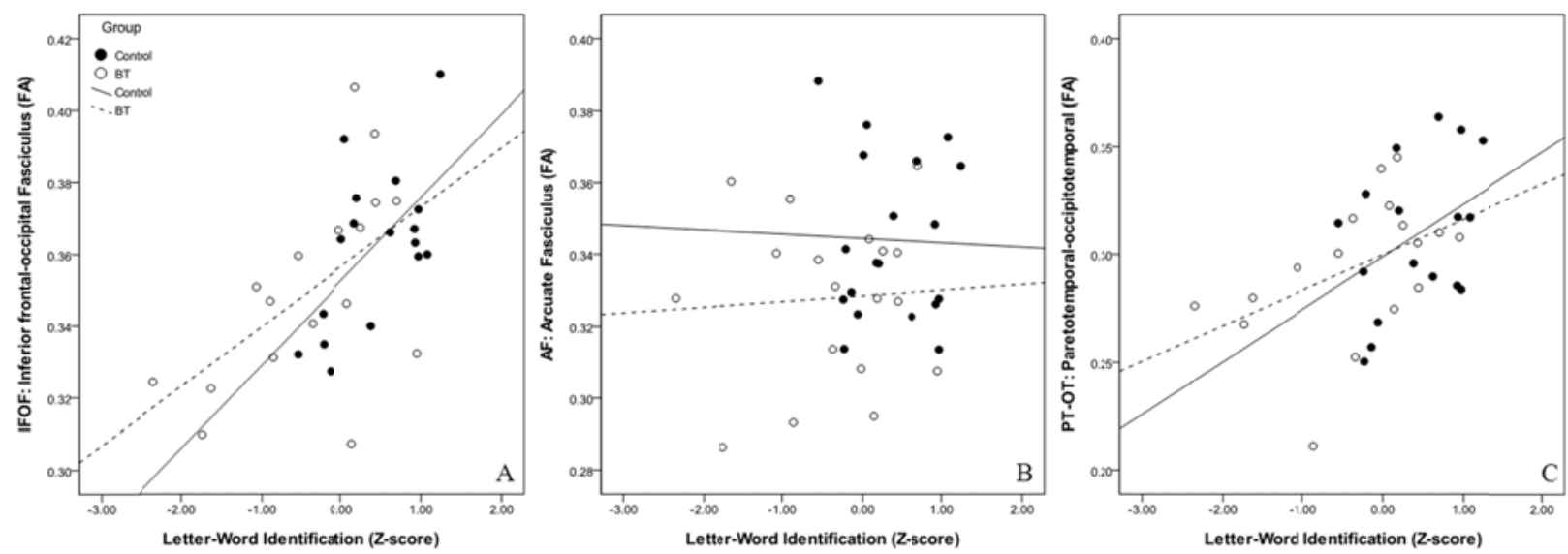

Figure 2. Tract microstructure (FA) and word reading by group. A. Inferior fronto-occipital fasciculus (IFOF) and word reading by group. Both survivors and control groups show a positive relationship between IFOF and word reading (controlling for education) reflected in $r$ values of 0.58 (control) and 0.45 (survivor). The large overlap between the groups highlights intact word reading in survivors. B. Arcuate fasciculus (AF) and word reading by group. Neither the survivor nor the control group show evidence for a relationship between FA in the Arcuate fasciculus and word reading. There appears to be less overlap between groups consistent with the significant group difference. C. Parietotemporal-Occipitotemporal (PT-OT) tract and word reading by group. Survivor and control participants show a similar positive relationship between FA in the PT-OT connection and word reading reflected in $\mathrm{r}$ values of 0.40 (control), and 0.46 (survivor). 


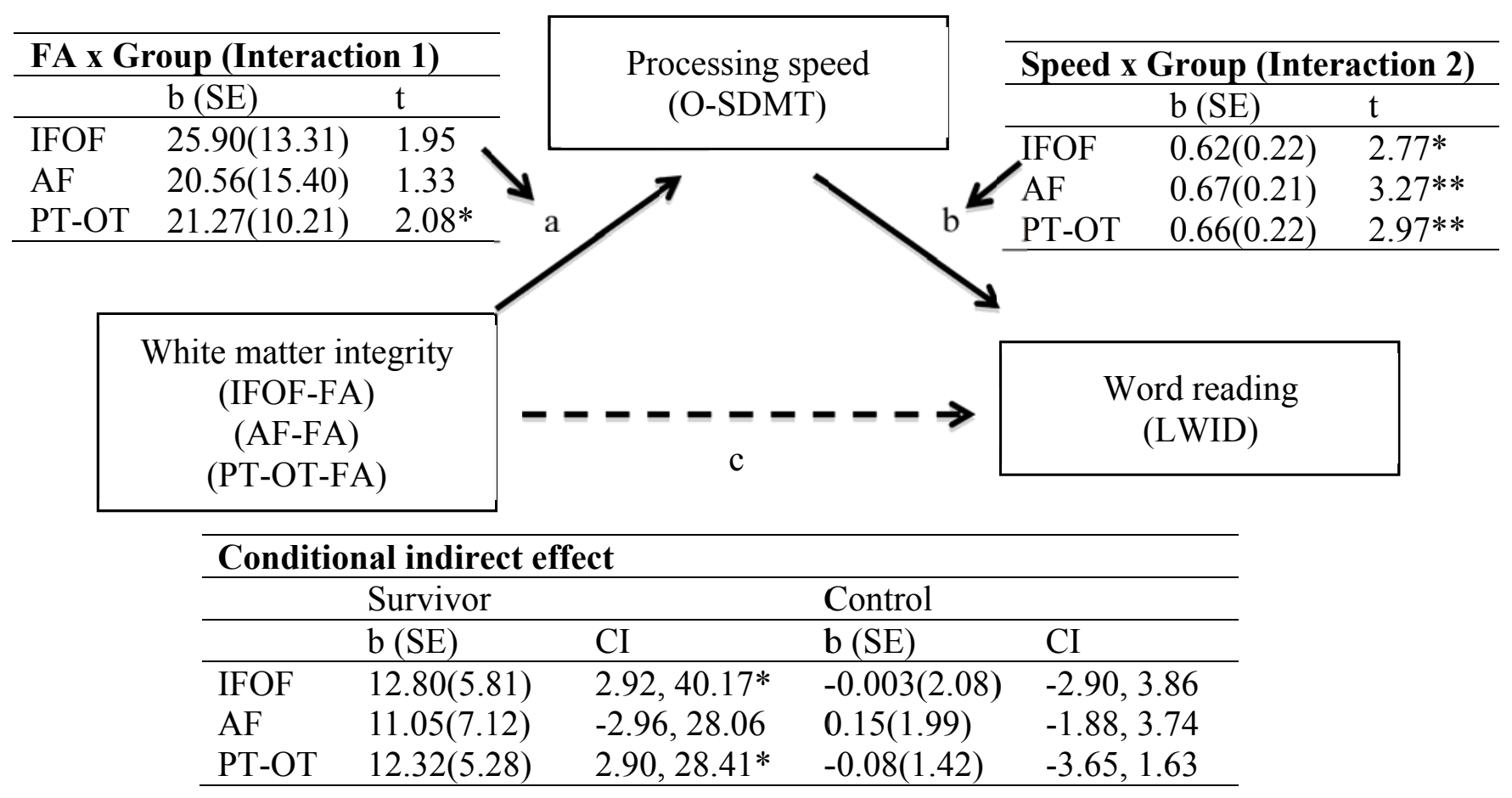

Figure 3. Resulting pathways of the moderated mediation model for each tract tested.

Confidence intervals indicate that the indirect effect of processing speed was conditional upon group with the survivor group showing a significant indirect effect for IFOF and PT-OT tracts. IFOF- Inferior fronto-occipital fasciculus, AF- Arcuate fasciculus, PT-OT- parietotemporaloccipitotemporal pathway, CI- confidence interval (bias corrected and accelerated). * indicates statistical significance at $\mathrm{p}<.05, * *$ indicates $\mathrm{p}<.01$. 